\title{
Application Research of BIM Technology in Green Building Construction
}

\author{
Liangli Xiao ${ }^{1, a}$, Zhuang Du ${ }^{1}$, Yan Liu ${ }^{1}$, Zhao Yang ${ }^{1}$, Kai Xu ${ }^{1}$ \\ ${ }^{1}$ Urban construction college, Wuhan University of Science and Technology, China
}

\begin{abstract}
Due to the complexity of the building and the comprehensiveness of multiple majors, a large number of uncertain factors, such as collision problems and construction schedule, often occur in the construction and lead to many resource waste problems which cannot be solved. The introduction of BIM technology into the construction of engineering projects can well overcome the collision in construction and complete the process of optimized construction schedule scheme through construction simulation to realize green building construction.
\end{abstract}

\section{Introduction}

The situation of high consumption and high pollution in traditional construction industry must be transformed into a sustainable development strategy mode that is resource conservation and environmental protection. How to meet the requirements and improve the production efficiency of the construction industry to achieve green building has become a common issue of the construction industry[1 3].

The significance of BIM technology, in the project cycle, is all construction building information that can share perfectly and communicate smoothly. It makes the project of each participation effectively work together throughout the project cycle. It fundamentally changes to use traditional 2D drawings for check, engineering construction and management of work mode.

Based on the specific project, the BIM technology is introduced into the structure construction through the establishment of the 3D model and integrated management platform to implement the dynamic input and output of information and real-time information modification. The BIM technology for collision analysis is aimed to find model conflict. Meanwhile the visual function is used to simulate the construction site and feedback construction progress the contractor in time. In this way, the simulation of site operation through the process of virtual construction can effectively control unnecessary waste in construction and save construction resources and period (see Figure1).

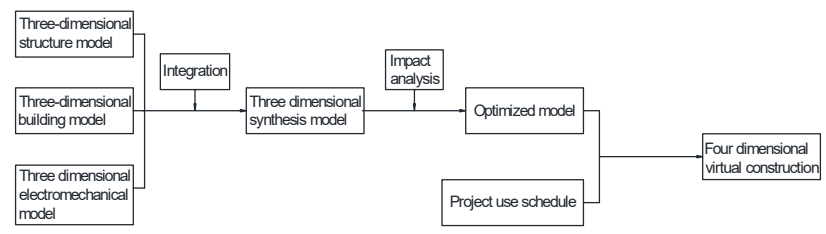

\section{Project overview}

This project is an office building with seven floors above ground, and the first layer height is $4.5 \mathrm{~m}$, others's is $3.7 \mathrm{~m}$. The upper part is steel member structure and the two underground layers are $4.5 \mathrm{~m}$ respectively, which are steel structure and concrete structure. The middle part of the office building is the main building. Both sides are skirt building, and there is a leisure area in the left skirt building.

Firstly it must complete the creation of the model with Revit and extract specific information through secondary development of the Revit API in the project, so it could make up disadvantages which the Revit cannot select multiple information at the same time. After that it can simulate the construction to provide information support. Then it analyses collisions with Navisworks between single professional and multiple professional. At the same time, it examines collision analysis with secondary development of Revit for single object and derives the results. Finally the optimized modeland construction schedule are connected to realize 4D construction progress of the simulation.

\section{Model establishment}

Each floor of this building structure is different and there are many interlayers in each floor. It is necessary to draw each layer, so it is more complicated to complete the structure drawing. Moreover, special attention should be paid to the placement and hole location of each beam when drawing. In this paper, the model is built with Revit from the first layer above ground, and then it is built the underground part.

Figure 1. Flowchart.

 


\subsection{Formation of whole model diagram and plan diagram}

Figure 2 shows the model of structure building after model establishment.

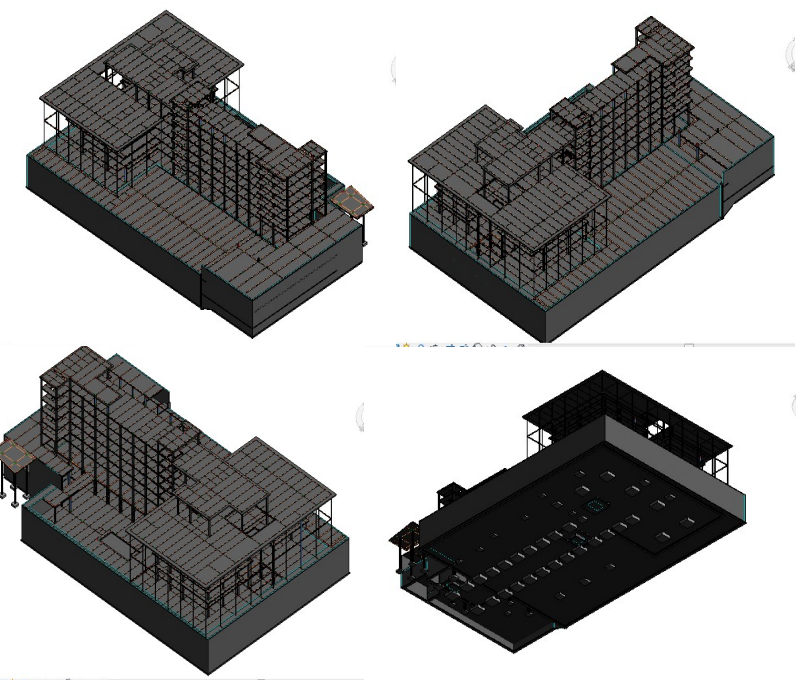

Figure 2. Angle diagram of three-dimensional model structure.

While drawing the model frame, it is also necessary to draw the internal steel structure of the concrete column. Therefore, we necessary to set steel protect layer thickness at first, as shown in Figure 3.

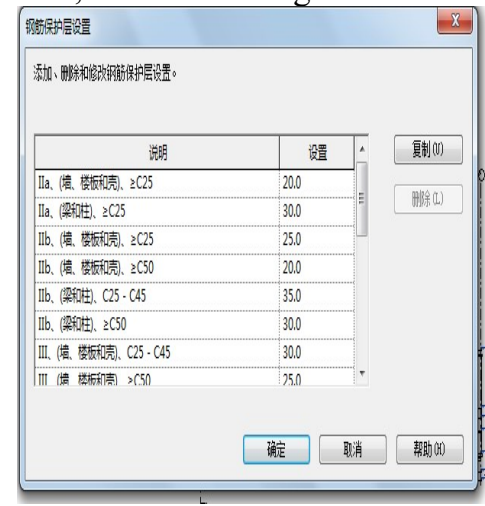

Figure 3. Setting of steel reinforcement protective layer.

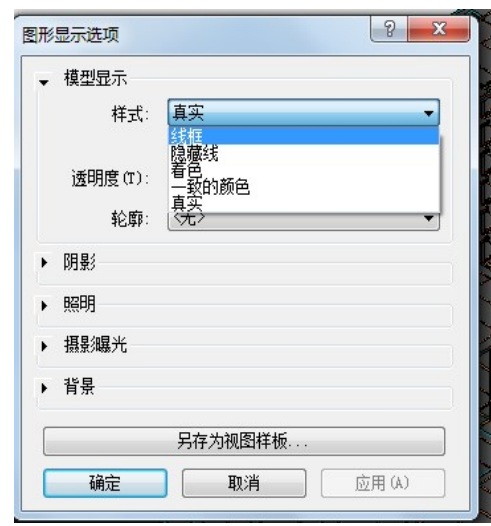

Figure 4. Graphical display box.

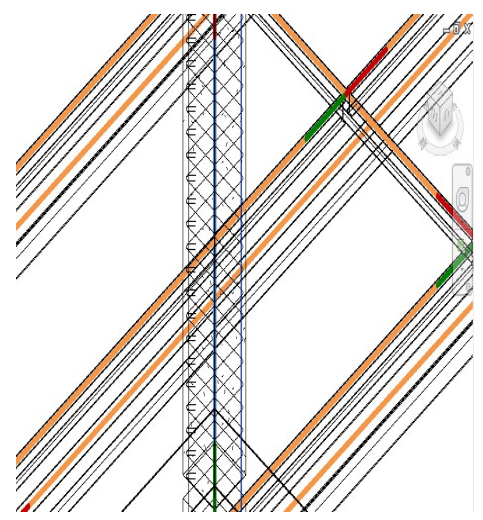

Figure 5. Steel Reinforcement Drawings of Concrete Columns.

Through the control chart Figure 4 of graphical display box can check the drawing of the internal reinforcement of the final model (see Figure 5). And the figure of the entire model can also be displayed (Figure 6).

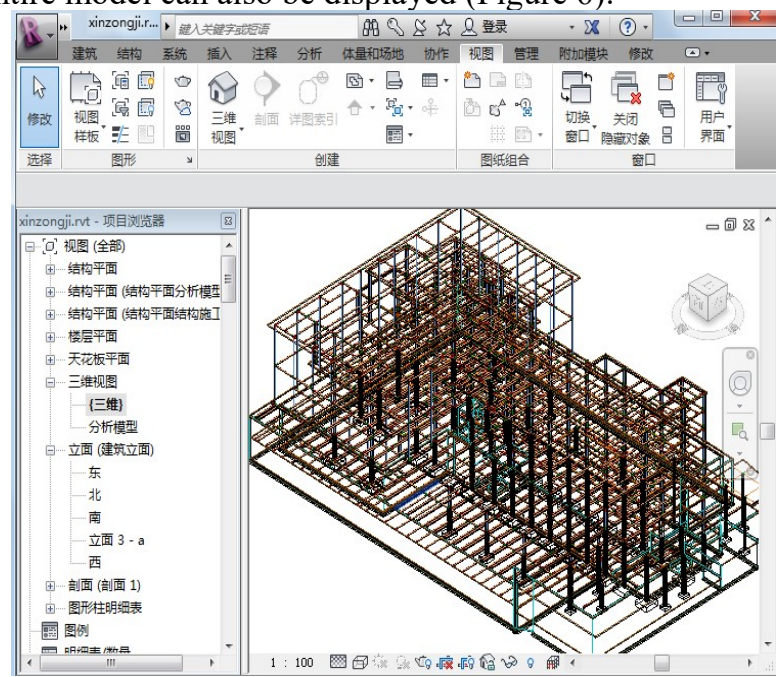

Figure 6. Model Wireframe.

The structure plan is the basic drawing used to reflect the structure. All other types of drawings need to be designed on the basis of the plan. Therefore, when using BIM technology to reflect the process of the structure, the expression of the plan must accurate and detail. So that conditions can be created to complete other drawings.

Through the selection of view visibility to model complete 2D drawing output. And this process is also the foundation that the designer convey work to the construction side. Figure 7 cut out part of the graph to show the basic plan.

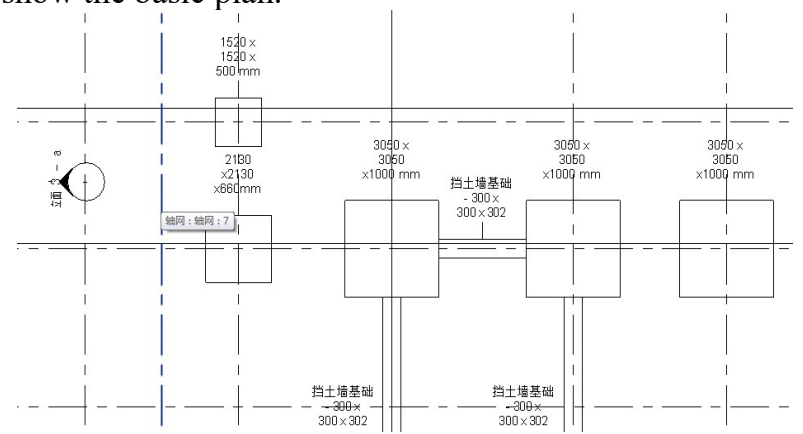

Figure 7. Basic plan. 


\subsection{Extraction of specific target data using API}

If the model need to construction simulation process, we should use the secondary development of Revit API to acquire component information in the model. Extraction process of information construction of the model, as shown in Figure 8 [4]:

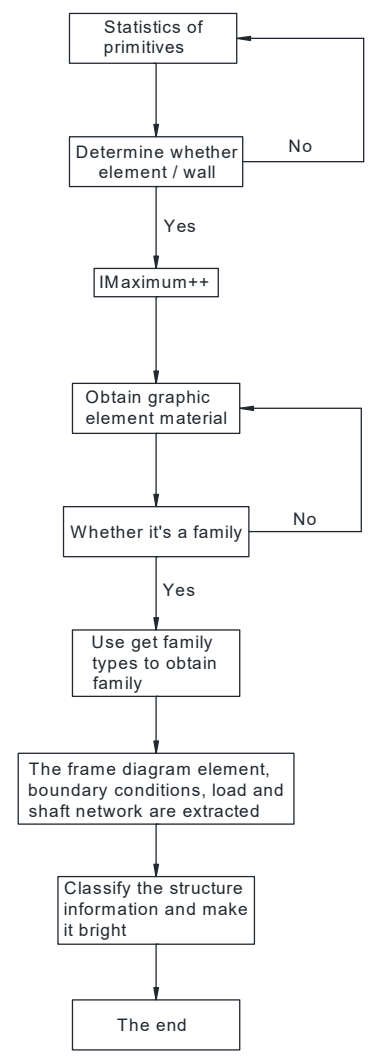

Figure 8. Development flow chart for specific data extraction.

Information resources that project need are obtained quickly through the information extraction process of the redevelopment project of Revit API. In order to provides a reliable basis for structural analysts to analyze the structure and provides component information for the virtual construction process.

\subsection{Model integration}

In the design, the drawings of the three specialty of architecture, structure and electromechanics engineering are completed by their respective corresponding departments. In order to conduct collision analysis, the models of the three majors must be integrated with each other. In Navisworks, it provides a good fusion of three professional models to combine several specialized models, as shown in Figure 9.
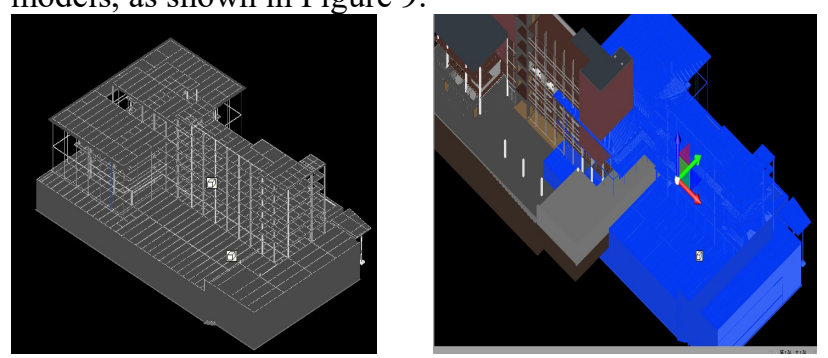

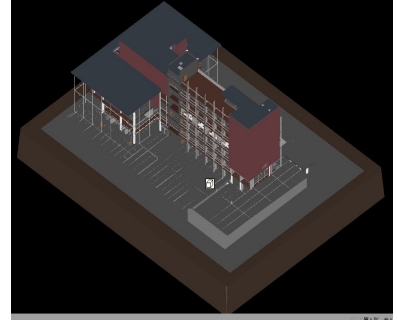

Figure 9. Model integration flow chart.

\section{Collision study in construction}

Navisworks is used for collision inspection. After model integration, collision analysis is carried out on the integrated model. Considering that too many collisions of the overall model are not conducive to post-collision inspection. Hence, it is divided into single-professional collision and inter-professional collision inspection.

\subsection{Single professional collision inspection}

For example, the model of single major is relatively simple to conduct collision analysis. The structural model only needs to be selected and adjusted to hard touch.

After selecting the items to be collided and run the test. The program will automatically pick the point where the collision occurs between the two sets of items and the result of the collision can be found (see Figure 10). In Figure 11, it can be seen that there are many collision points in the results. Each collision point can be associated with the corresponding collision position. Then the verification of each collision point can be made gradually to confirm whether each collision point is true or not and whether the collision can be ignored. Where collisions are found, you can return to the Revit model for modifications (see Figure 12).

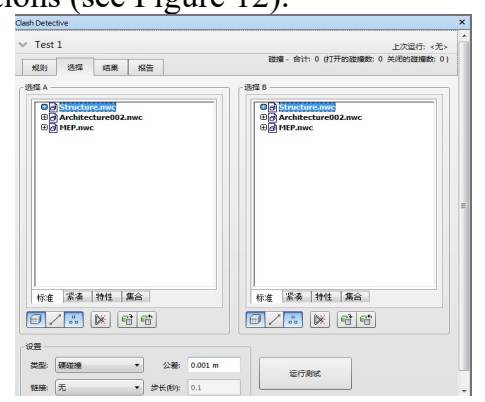

Figure 10. Single professional collision selection diagram.

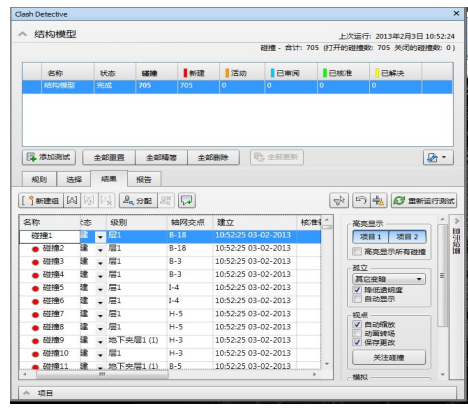

Figure 11. Collision results. 


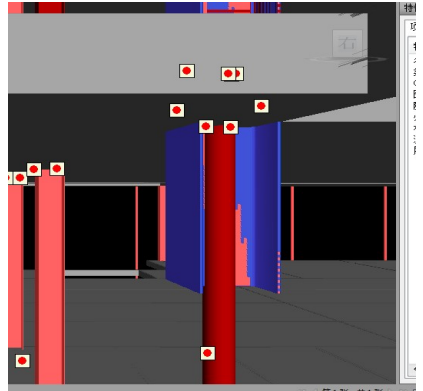

Figure 12. Collision point diagram.

\subsection{Collision detection between different majors}

Multi-professional comprehensive collisions include electromechanical and collisions with structures and buildings. Two points should be paid attention to achieve accurate and quick analysis. First of all, the number of entities inside a building's pipelines is large and the arrangement is complicated. If all collision are detected, the result will have too much and very confusing. It is difficult to perform statistical and can lead to a computer running speed is slow. In order to get a better display effect, so under the goal of completing the function, the number of entities should be minimized. Then the test is generally a floor.

After the search set is defined, the corresponding set can be selected in the selection project. According to the type of each model, multiple selection sets are created for the model and then the model is collided. Considering that the electromechanical model of this model is relatively empty, electromechanical model is used as a whole to collide with other management sets. Such as the collision between structural column and electromechanical model (see Figure 13). According to the collision analysis of structural column and electromechanical model, the following collision results and corresponding graphs can be obtained (see Figure 14).

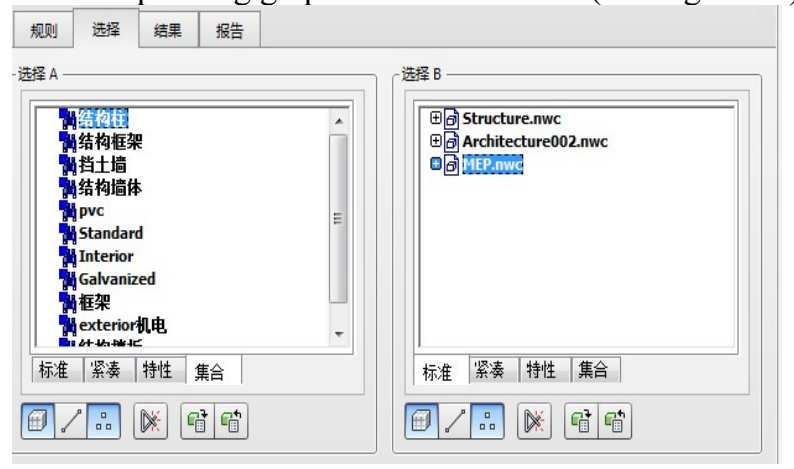

Figure 13. Collision cell selection diagram.

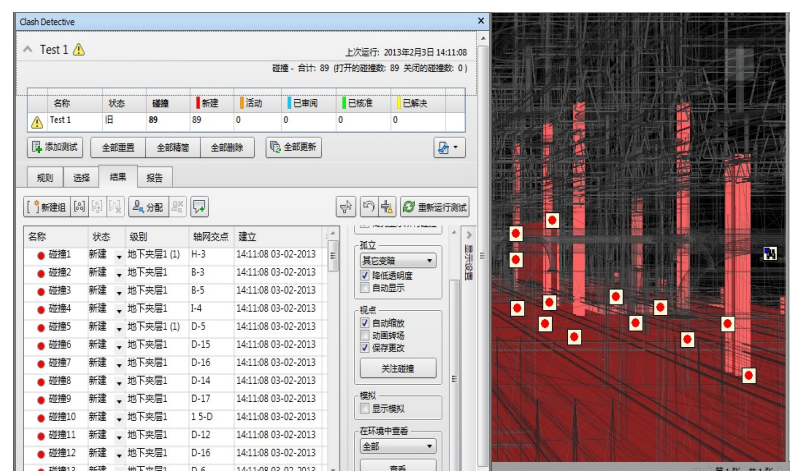

Figure 14. Schematic diagram of electromechanical model and structural column collision.

When collision occurs, check collision points can also add comments at collision points. It is beneficial to the allocation and inspection of collision points and assign each collision point to the responsible design department.

\subsection{Secondary development of component collision detection using API}

In the collision process under the construction of Navisworks, it is mainly the collision process of each model or between each other. If the collision phenomenon of a single component is needed, it may be difficult to be satisfied. In Visual Studio, Revit and secondary software development can be carried out, through API interface. It can correspond to the collision detection of a single object.

\subsection{Construction simulation on 4D}

After the collision analysis process in the project construction is completed. the optimized model after the collision problem is solved shall be used for the four dimensional virtual construction process so as to modify the construction scheme and construction schedule in the project process. In order to complete the whole project better.

\subsubsection{Use MS project software to compile the construction schedule}

1) Project decomposition: according to the subdivision of the building model, the project is decomposed into WBS coding.

2) According to the model of the Project, the Project software is used to compile the reasonable construction process and movement relationship of the model components. so as to compile a more detailed schedule plan and form Gantt chart, as shown in Figure 15-16. 


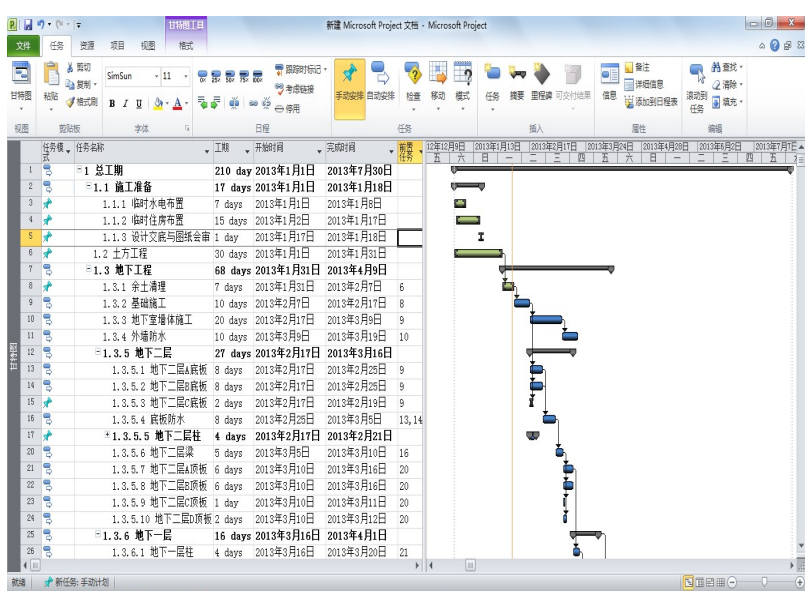

Figure 15. Gantt chart of project construction progress.

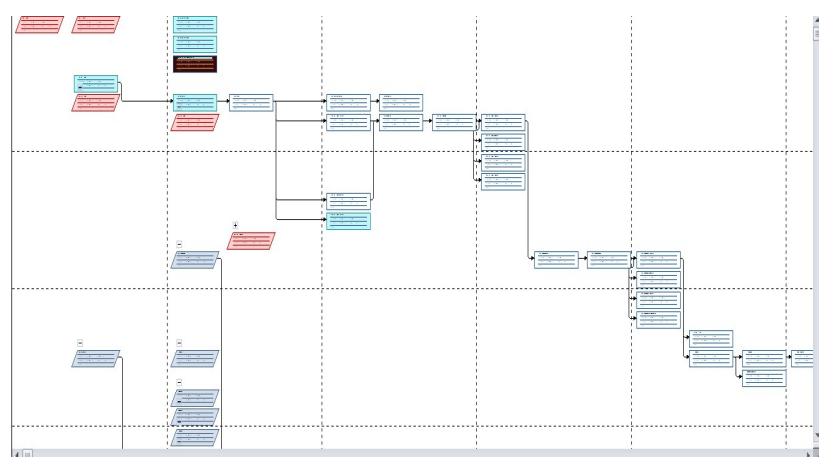

Figure 16. Construction network planning network diagram.

\subsubsection{Simulation of construction schedule}

Import Navisworks from the data source (as shown in Figure 17), according to the construction schedule that has been prepared using the Project software. Before the start of the actual engineering project, virtual construction are demonstrated on the computer. which can be found and avoid may arise in the actual engineering situation, and change the plan or prevent it. It is advantageous to the solution of optimization design and improve the efficiency of the construction project construction. To avoid the unnecessary loss of resources and achieve the green construction[5 7].

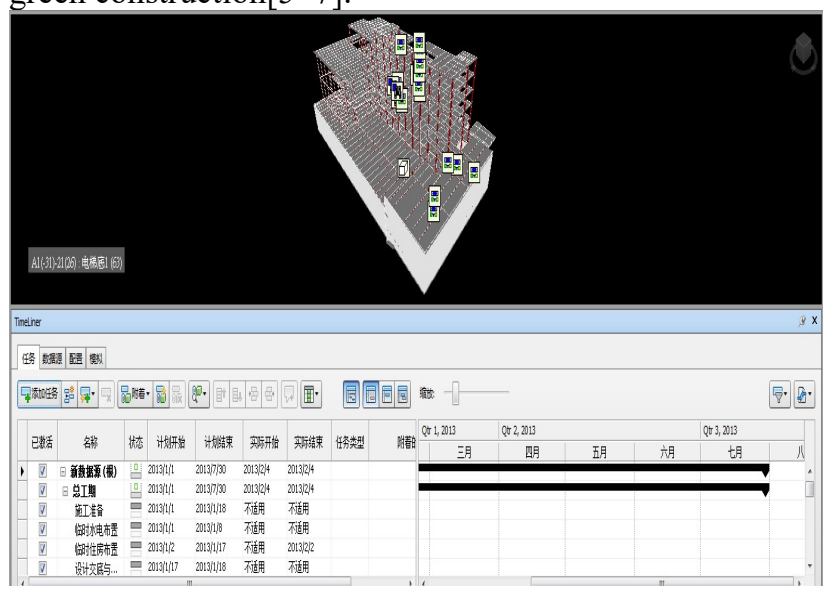

Figure 17. Progress plan data source import diagram.

As shown in Figure 17, the Gantt hart will appear on the right side of the task bar after the task is imported.
Since there is no correlation between the construction schedule and model construction, the corresponding correlation should be made. After a series of work before virtual construction is completed, the process of virtual construction begins. Construction units can see, in different period, the whole floor construction and completion of each task according to task bar text. And through the observation of the specified date or specified weeks, it can be seen the whole project completion in the current date (see Figure 18). So that you can see the time limit for a project is delay or advance with the actual engineering. It helps to master the progress in the construction and have specific understanding of the whole project.

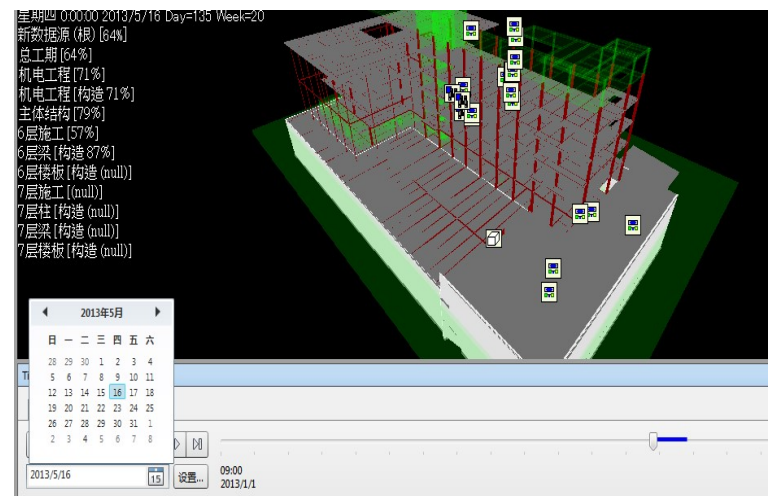

Figure 18. Comparison diagram of actual project and virtual progress.

\section{Conclusion}

1) The establishment of the model and the secondary development of Revit API can show the advantages of Revit modeling. The secondary development of API can further strengthen the control of the modeling information and complete the extraction process of project information more conveniently and quickly. It make the information of the model more clear, which is conducive to strengthening the analysis ability of the structure.

2) The inspection analysis of the collision in the model construction can not only quickly check out the various collision problem of model, but also can be very intuitive to see collision point from the perspectives of the $3 \mathrm{D}$ and annotate the collision problem. The classification is handed over to the corresponding design unit, which solves the collision problem more quickly and completes the cooperation between the various professions.

3) The 4D virtual construction schedule based on BIM technology can simulate the implementation of the project in the virtual environment. Through the construction process simulation to discover problems and timely solve problems so as to achieve the optimized effect and guide the construction of the project, providing strong guarantee for the smooth completion of the project. 


\section{References}

1. Li Haiming, Application of BIM technical system standard in green public building design $[\mathrm{J}]$. CAS. 11, (2016).

2. Gu Jinrong, Application of BIM technology in green public building design [J]. Jiangxi building materials. 13, (2017).

3. Zhang Li, Application of BIM technology in green public building design [J]. Building materials and decoration. 19, (2016).

4. Liu Shihua, application research of BIM based virtual construction technology [D]. master's thesis of Xi'an university of architecture and technology,65-70 (2012).
5. Xu Xiaoli, Application of BIM technology in engineering project construction $[\mathrm{J}]$. New technology and new products in China. 9, (2018).

6. Huang Cheng, Yang Huanyun, Peng Hexin, Gao changgui, Pan Jiaqi. Simple analysis of application of BIM technology in construction simulation optimization $[\mathrm{J}]$. Enterprise technology and development. 3, (2018).

7. Chen Huiqun. Analysis and research on the application of BIM technology in construction $[\mathrm{J}]$. Residential industry. 5, (2018). 